\title{
Postnikov invariants of H-spaces
}

\author{
by \\ Dominique A r lettaz (Lausanne) and \\ Nicole Pointet-Tischler (Bonn)
}

\begin{abstract}
It is known that the order of all Postnikov $k$-invariants of an $\mathrm{H}$-space of finite type is finite. This paper establishes the finiteness of the order of the $k$-invariants $k^{m+1}(X)$ of $X$ in dimensions $m \leq 2 n$ if $X$ is an $(n-1)$-connected H-space which is not necessarily of finite type $(n \geq 1)$. Similar results hold more generally for higher $k$-invariants if $X$ is an iterated loop space. Moreover, we provide in all cases explicit universal upper bounds for the order of the $k$-invariants of $X$.
\end{abstract}

Introduction. The Postnikov invariants of a connected simple CWcomplex $X$ are cohomology classes which provide the necessary information for the reconstruction of $X$, up to weak homotopy equivalence, from its homotopy groups. More precisely, let $\alpha_{m}: X \rightarrow X[m]$ denote the $m$ th Postnikov section of $X$ for any positive integer $m$, i.e., $X[m]$ is a CWcomplex obtained from $X$ by adjoining cells of dimensions $\geq m+2$ such that $\pi_{i} X[m]=0$ for $i>m$ and $\left(\alpha_{m}\right)_{*}: \pi_{i} X \rightarrow \pi_{i} X[m]$ is an isomorphism for $i \leq m$. The Postnikov $k$-invariants of $X$ are cohomology classes $k^{m+1}(X) \in$ $H^{m+1}\left(X[m-1] ; \pi_{m} X\right)$ with the property that $X[m]$ is homotopic to the fibre of the map $X[m-1] \rightarrow K\left(\pi_{m} X, m+1\right)$ corresponding to $k^{m+1}(X)$, for $m \geq 2$ (see for instance [WG2], Section IX.2).

A classical result of M. Arkowitz and C. Curjel (see [AC], Proposition 4.1, and $[\mathrm{T}]$, Théorème 6 ) asserts that if $X$ is an H-space of finite type, then all its $k$-invariants are cohomology classes of finite order. The present paper is devoted to the following two questions:

(A) Is it still true that the $k$-invariants of an $\mathrm{H}$-space $X$ have finite order if $X$ is not of finite type?

1991 Mathematics Subject Classification: Primary 55S45; Secondary 55P35, 55P45.

The main results of this paper are contained in the second author's Ph.D. thesis [P1]. The second author would like to thank the Swiss National Science Foundation for financial support and the Max-Planck-Institut für Mathematik in Bonn for hospitality. 
(B) Is it possible to approximate the order of the $k$-invariants of $\mathrm{H}$-spaces in a universal way?

Of course, both questions are related: an affirmative answer to Question (B) would imply that Question (A) also has a positive answer. In other words, if there is an upper bound $A(m)$, depending only on $m$, for the order of $k^{m+1}(X)$ for all $\mathrm{H}$-spaces of finite type $X$, then a direct limit argument shows that $A(m) k^{m+1}(X)=0$ even if $X$ is an $\mathrm{H}$-space which is not of finite type.

Partial results on that problem have been previously obtained. C. Soulé proved that if $X$ is an $(n-1)$-connected $\mathrm{H}$-space of finite type with $n \geq 2$, then the $k$-invariant $k^{m+1}\left(X_{(p)}\right)$ of the localization of $X$ at the prime $p$ vanishes if $m \leq n+2 p-4$ (see [So], proof of Proposition 3, where the argument uses an idea of [Sm]). The first author established in [A2], Theorem 1.6, and [A4], Theorem 1.3, the existence of universal upper bounds for the order of the $k$-invariants in the stable range, i.e., for the $k$-invariants $k^{m+1}(X)$ of $(n-1)$-connected $r$-fold loop spaces $X$ assuming that $m \leq 2 n+r-2$ (see Definition 4.4 and Theorem 4.5 below). In the special case where $r=2$, $n=1, m=2$, he was actually able to show that $k^{3}(X)=0$ for all connected double loop spaces $X$ (see [A3], Theorem A).

This paper is devoted to the solution of the problem given by Questions $\mathrm{A}$ and $\mathrm{B}$ in the non-stable case. Our first result determines universal upper bounds for the order of the first $k$-invariant of an H-space (see Corollary 3.3). Its proof is based on the study of the cohomology suspension for EilenbergMacLane spaces.

The first $k$-invariant $k^{n+2}(X) \in H^{n+2}\left(K\left(\pi_{n} X, n\right) ; \pi_{n+1} X\right)$ of any $(n-1)$-connected $H$-space $X$ (with $n \geq 1)$ fulfills

$$
2 k^{n+2}(X)=0 \text {. }
$$

We are then able to extend this to some $k$-invariants of $\mathrm{H}$-spaces and more generally to all higher $k$-invariants of iterated loop spaces. More precisely, we define, for $r \geq 1$, positive integers $C_{r}(m, n)$, depending only on $m, n$ and $r$, and prove the following theorems (see Corollary 4.8, Theorems 4.10 and 4.11).

For any $(n-1)$-connected $H$-space $X($ with $n \geq 1)$ and any integer $m$ such that $n+1 \leq m \leq 2 n$, the $k$-invariant $k^{m+1}(X)$ satisfies

$$
C_{1}(m, n) k^{m+1}(X)=0 \quad \text { in } H^{m+1}\left(X[m-1] ; \pi_{m} X\right) .
$$

For any $(n-1)$-connected $r$-fold loop space $X$ (with $n \geq 1$ and $r \geq 2)$ and any integer $m$ such that $n+1 \leq m \leq(r+1) n+r$, the $k$-invariant $k^{m+1}(X)$ satisfies

$$
C_{r}(m, n) k^{m+1}(X)=0 \quad \text { in } H^{m+1}\left(X[m-1] ; \pi_{m} X\right) .
$$


In other words, we get an upper bound for the order of the $k$-invariant $k^{m+1}(X)$ of an $(n-1)$-connected CW-complex $X$ if $X$ is an $\left[\frac{m}{n+1}\right]$-fold loop space, where $\left[\frac{m}{n+1}\right]$ denotes the integral part of $\frac{m}{n+1}$, except for the case $m=2 n+1$ where $X$ needs to be a double loop space (see Corollary 4.12). Observe that we always consider H-spaces or iterated loop spaces which are not necessarily of finite type, and that all upper bounds we obtain are universal in the sense that they only depend on the dimension of the $k$ invariant we are looking at and on the connectivity of the space, but not on the space itself.

As an application, we then approximate the order of the identity of Postnikov sections of homotopy associative $\mathrm{H}$-spaces and iterated loop spaces. We obtain the following results (see Corollaries 5.5 and 5.6).

Let $X$ be an $(n-1)$-connected homotopy associative $H$-space (with $n \geq 1)$, $m$ an integer satisfying $n+1 \leq m \leq 2 n$, and assume that $\pi_{i} X$ has finite exponent $s_{i}$ for $n \leq i \leq m$. Then the order of the identity of $X[m]$ divides the product

$$
\operatorname{lcm}\left(s_{n}, \ldots, s_{m}\right) \prod_{i=n+1}^{m} C_{1}(i, n) .
$$

Let $X$ be an $(n-1)$-connected $r$-fold loop space (with $n \geq 1$ and $r \geq 2), m$ an integer satisfying $n+1 \leq m \leq(r+1) n+r$, and assume that $\pi_{i} X$ has finite exponent $s_{i}$ for $n \leq i \leq m$. Then the order of the identity of $X[m]$ divides the product

$$
\operatorname{lcm}\left(s_{n}, \ldots, s_{m}\right) \prod_{i=n+1}^{m} C_{r}(i, n) .
$$

The paper is organized as follows. Section 1 presents the main properties of spaces with $k$-invariants of finite order. In Section 2, we recall from [P2] that the exponent of the image of the cohomology suspension for products of Eilenberg-MacLane spaces is bounded in a universal way. Section 3 provides the solution of the above problem for the first $k$-invariant of an $\mathrm{H}$-space. We prove our main results on high-dimensional $k$-invariants of H-spaces and iterated loop spaces in Section 4. Finally, Section 5 is devoted to the study of the order of the identity.

Throughout the paper, we use the following notation. For an abelian group $G$ and a positive integer $n, K(G, n)$ denotes the Eilenberg-MacLane space having all homotopy groups trivial except for $G$ in dimension $n$. If $X$ is an $(n-1)$-connected CW-complex (with $n \geq 1$ ) and if $m$ and $i$ are integers such that $n-1 \leq i<m$, we write $\alpha_{m}: X \rightarrow X[m]$ for the $m$ th Postnikov section of $X$ and $X(i, m]$ for the $i$ th connected cover of $X[m]$. 
1. $k$-invariants of finite order. If $X$ is a connected simple CWcomplex, it is well known that the vanishing of the $k$-invariant $k^{m+1}(X) \in$ $H^{m+1}\left(X[m-1] ; \pi_{m} X\right)$ is equivalent to the fact that the $m$ th Postnikov section $X[m]$ of $X$ is a product $X[m] \simeq X[m-1] \times K\left(\pi_{m} X, m\right)$, and to the split injectivity of the Hurewicz homomorphism $h_{m}: \pi_{m} X \rightarrow H_{m}(X ; \mathbb{Z})$. This can be generalized when the $k$-invariant $k^{m+1}(X)$ is a cohomology class of finite order in the group $H^{m+1}\left(X[m-1] ; \pi_{m} X\right)$.

Proposition 1.1. Let $X$ be a connected simple $C W$-complex, $m$ an integer $\geq 2$ and $\varrho$ a positive integer. The following assertions are equivalent:

(a) $\varrho k^{m+1}(X)=0$ in $H^{m+1}\left(X[m-1] ; \pi_{m} X\right)$.

(b) There is a map $f_{m}: X \rightarrow K\left(\pi_{m} X, m\right)$ such that the induced homomorphism $\left(f_{m}\right)_{*}: \pi_{m} X \rightarrow \pi_{m} X$ is multiplication by $\varrho$.

(c) There is a homomorphism $\theta_{m}: H_{m}(X ; \mathbb{Z}) \rightarrow \pi_{m} X$ such that the composition

$$
\pi_{m} X \stackrel{h_{m}}{\longrightarrow} H_{m}(X ; \mathbb{Z}) \stackrel{\theta_{m}}{\longrightarrow} \pi_{m} X
$$

is multiplication by $\varrho$.

Proof. Lemma 4 of [A1] (see also Lemma 1.4 of [A4]) shows that (a) implies (b). Assertion (c) follows from (b) because of the commutativity of the diagram

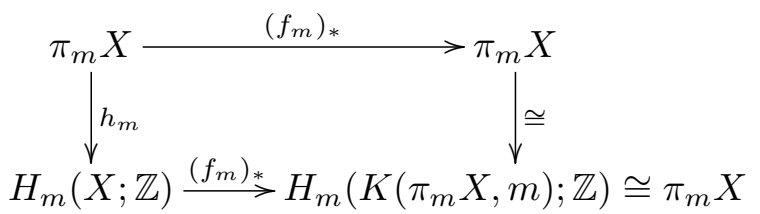

induced by the map $f_{m}$, where both vertical arrows are Hurewicz homomorphisms: we let $\theta_{m}$ be the bottom horizontal homomorphism $\left(f_{m}\right)_{*}$ in that diagram.

In order to prove that (a) follows from (c), let us recall the definition of the $k$-invariants of a connected simple CW-complex $X$ (see [WG2], Section IX.2). Let $\kappa^{m+1}$ denote the composition

$$
H_{m+1}(X[m-1], X[m] ; \mathbb{Z}) \stackrel{\left(\bar{h}_{m+1}\right)^{-1}}{\longrightarrow} \pi_{m+1}(X[m-1], X[m]) \stackrel{\partial}{\rightarrow} \pi_{m} X,
$$

where $\bar{h}_{m+1}$ is the Hurewicz isomorphism for the $m$-connected pair $(X[m-1]$, $X[m]$ ) and $\partial$ the composition of the connecting homomorphism (which is actually an isomorphism) of the homotopy exact sequence of that pair with the obvious isomorphism $\pi_{m} X[m] \cong \pi_{m} X$. Consider the isomorphism

$$
\lambda: \operatorname{Hom}\left(H_{m+1}(X[m-1], X[m] ; \mathbb{Z}), \pi_{m} X\right) \stackrel{\cong}{\rightrightarrows} H^{m+1}\left(X[m-1], X[m] ; \pi_{m} X\right)
$$

given by the universal coefficient theorem and the homomorphism

$$
\mu: H^{m+1}\left(X[m-1], X[m] ; \pi_{m} X\right) \rightarrow H^{m+1}\left(X[m-1] ; \pi_{m} X\right)
$$


induced by the inclusion of pairs $(X[m-1], *) \hookrightarrow(X[m-1], X[m])$. The $k$-invariant $k^{m+1}(X)$ is defined by

$$
k^{m+1}(X)=\mu \lambda\left(\kappa^{m+1}\right) \in H^{m+1}\left(X\left[m-1 \mid ; \pi_{m} X\right) .\right.
$$

Now let us look at the commutative diagram

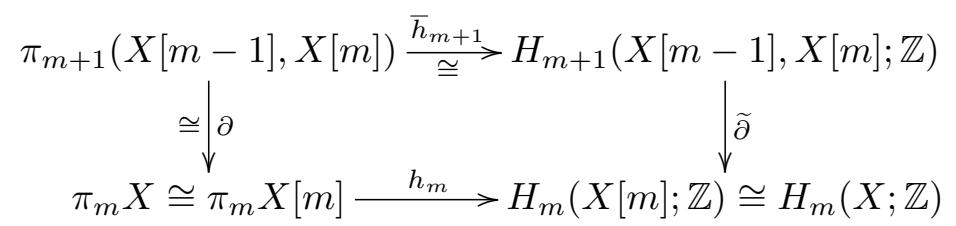

in which the horizontal arrows are Hurewicz homomorphisms and the vertical arrows are connecting homomorphisms. If $\theta_{m}: H_{m}(X ; \mathbb{Z}) \rightarrow \pi_{m} X$ exists as in (c), we deduce that

$$
\theta_{m} \widetilde{\partial}=\theta_{m} h_{m} \partial\left(\bar{h}_{m+1}\right)^{-1}=\varrho \partial\left(\bar{h}_{m+1}\right)^{-1}=\varrho \kappa^{m+1}
$$

and that $\varrho \lambda\left(\kappa^{m+1}\right)$ belongs to the image of the connecting homomorphism $\delta: H^{m}\left(X[m] ; \pi_{m} X\right) \rightarrow H^{m+1}\left(X[m-1], X[m] ; \pi_{m} X\right)$. The exactness of the cohomology sequence

$$
\begin{aligned}
H^{m}\left(X[m] ; \pi_{m} X\right) \stackrel{\delta}{\rightarrow} H^{m+1}(X[m-1], X[m] ; & \left.\pi_{m} X\right) \\
& \stackrel{\mu}{\rightarrow} H^{m+1}\left(X[m-1] ; \pi_{m} X\right)
\end{aligned}
$$

of the pair $(X[m-1], X[m])$ finally implies $\varrho k^{m+1}=\varrho \mu \lambda\left(\kappa^{m+1}\right)=0$.

If $X$ is a homotopy associative $\mathrm{H}$-space, the finiteness of the order of its $k$-invariants has another important consequence.

Proposition 1.2. Let $X$ be a connected homotopy associative H-space, $m$ an integer $\geq 2$, and assume that $k^{i+1}(X)$ is of finite order dividing $\varrho_{i}(X)$ for $2 \leq i \leq m$. Then there are maps

$$
\varphi_{m}: X[m] \rightarrow \prod_{i=1}^{m} K\left(\pi_{i} X, i\right) \quad \text { and } \quad \psi_{m}: \prod_{i=1}^{m} K\left(\pi_{i} X, i\right) \rightarrow X[m]
$$

such that the composition $\psi_{m} \varphi_{m}: X[m] \rightarrow X[m]$ is homotopic to the @th power map, where @ denotes the product $\prod_{i=2}^{m} \varrho_{i}(X)$.

Proof. The assertion follows from the argument of the proof of Corollary 1.4 of [A5], where the same statement is formulated for spectra.

Because of all these nice properties, it is of particular interest to find finiteness results for the order of the $k$-invariants of certain $\mathrm{CW}$-complexes and to be able to approximate their order: this is the goal of the remainder of the present paper. Let us conclude this section by mentioning a surprising observation which illustrates one difference between CW-complexes of finite type and those which are not of finite type. 
REMARK 1.3. The fact that a $\mathrm{CW}$-complex $X$ is rationally a product of Eilenberg-MacLane spaces (in other words, that the $k$-invariants of the rationalization of $X$ are trivial) does not imply that the $k$-invariants of $X$ have finite order. Consider the following example. If $n$ is an even integer, then $H_{2 n+1}(K(\mathbb{Q}, n) ; \mathbb{Z})=0$ and $H_{2 n}(K(\mathbb{Q}, n) ; \mathbb{Z}) \cong \mathbb{Q}$. Thus, the universal coefficient theorem shows that

$$
H^{2 n+1}(K(\mathbb{Q}, n) ; \mathbb{Z}) \cong \operatorname{Ext}\left(H_{2 n}(K(\mathbb{Q}, n) ; \mathbb{Z}), \mathbb{Z}\right) \cong \operatorname{Ext}(\mathbb{Q}, \mathbb{Z}) \cong \mathbb{R} .
$$

Now, choose a non-trivial element $\alpha$ in $H^{2 n+1}(K(\mathbb{Q}, n) ; \mathbb{Z})$ and denote by $X$ the fibre of the corresponding map

$$
\alpha: K(\mathbb{Q}, n) \rightarrow K(\mathbb{Z}, 2 n+1) .
$$

The CW-complex $X$ has only two non-trivial homotopy groups, $\pi_{n} X \cong \mathbb{Q}$ and $\pi_{2 n} X \cong \mathbb{Z}$, and its only $k$-invariant is $k^{2 n+1}(X)=\alpha$, which is of infinite order. However, the rationalization $X_{\mathbb{Q}}$ of $X$ has again two nontrivial homotopy groups $\pi_{n} X_{\mathbb{Q}} \cong \pi_{2 n} X_{\mathbb{Q}} \cong \mathbb{Q}$ and its $k$-invariant $k^{2 n+1}\left(X_{\mathbb{Q}}\right)$ is trivial since it belongs to the group

$$
H^{2 n+1}(K(\mathbb{Q}, n) ; \mathbb{Q}) \cong \operatorname{Ext}(\mathbb{Q}, \mathbb{Q})=0 .
$$

Consequently, $X_{\mathbb{Q}}$ is a product of Eilenberg-MacLane spaces:

$$
X_{\mathbb{Q}} \simeq K(\mathbb{Q}, n) \times K(\mathbb{Q}, 2 n) .
$$

It is however true that if $X$ is a CW-complex of finite type which is rationally a product of Eilenberg-MacLane spaces, then all $k$-invariants of $X$ have finite order.

2. The cohomology suspension. The main results on $k$-invariants of (iterated) loop spaces we obtain in this paper rely on the following basic property: the $k$-invariants of a loop space are cohomology classes which belong to the image of the cohomology suspension. More precisely, if $Y$ is a simply connected CW-complex and $m$ an integer $\geq 3$, then the $k$-invariant $k^{m+1}(Y) \in H^{m+1}\left(Y[m-1] ; \pi_{m} Y\right)$ of $Y$ and the $k$-invariant $k^{m}(\Omega Y)$ of $\Omega Y$, which is in fact a cohomology class in $H^{m}\left(\Omega Y[m-2] ; \pi_{m} Y\right)$ since $\pi_{m-1}(\Omega Y) \cong \pi_{m} Y$, are related by the following formula (see [WG2], p. 438).

Proposition 2.1. Let $Y$ be a simply connected $C W$-complex and $m$ an integer $\geq 3$. If we denote by $\sigma^{*}$ the cohomology suspension

$$
H^{n+1}\left(Y[n-1] ; \pi_{n} Y\right) \rightarrow H^{n}\left(\Omega Y[n-2] ; \pi_{n} Y\right),
$$

then $\sigma^{*}\left(k^{n+1}(Y)\right)=k^{n}(\Omega Y)$.

The goal of this section is to recall a result from [P2] on the cohomology suspension of a finite product of Eilenberg-MacLane spaces in unstable dimensions. It asserts that the exponent of its image is finite and can be estimated by a universal upper bound. This upper bound only depends on 
the dimension and the connectivity of the space, and not on its homotopy groups. Although $k$-invariants do not belong in general to the cohomology of a product of Eilenberg-MacLane spaces except the first non-trivial one, we will show in Section 4 how this theorem can provide universal upper bounds for the order of higher $k$-invariants of $\mathrm{H}$-spaces and iterated loop spaces.

Definition 2.2 (see [P2]). For integers $m>n \geq 1$ and a prime $q$, let

$$
\varphi(q, m, n)=\max \left\{1,\left[\log _{q} \frac{m}{n-2+2 q}\right]+1\right\},
$$

where [-] denotes the integral part. This allows one to define integers $S_{1}(m, n)$ and $S_{2}(m, n)$ by

$$
S_{1}(m, n)=\prod_{\substack{q \leq(m-n) / 2+1 \\ q \text { prime }}} q^{\varphi(q, m, n)}, \quad S_{2}(m, n)=\prod_{\substack{q \leq(m-n) / 2+1 \\ q \text { prime }}} q^{\varphi(q, m, n)+1},
$$

and to set

$$
B(m, n)=S_{1}(m+1, n+1) S_{2}(m+1, n+1) S_{2}(m, n+1)
$$

for $m>n \geq 1$.

Notice that a prime $p$ divides $B(m, n)$ if and only if $p \leq(m-n) / 2+1$.

We are now able to state the main theorem of [P2].

Theorem 2.3. Let $I$ be a finite set of positive integers, $n=\min I$ and $m>\max I$. Then for any family $\left\{G_{i}\right\}_{i \in I}$ of abelian groups and any abelian group $H$, the cohomology suspension

$$
\sigma^{*}: H^{m+2}\left(\prod_{i \in I} K\left(G_{i}, i+1\right) ; H\right) \rightarrow H^{m+1}\left(\prod_{i \in I} K\left(G_{i}, i\right) ; H\right)
$$

satisfies $B(m+1, n) \operatorname{im}\left(\sigma^{*}\right)=0$.

The proof of this result is given in [P2] and [P1], Chapitres 2 and 3. It is essentially based on Cartan's description of the Pontryagin algebra $H_{*}(K(G, n) ; \mathbb{Z})$ where $G$ is an abelian group and $n \geq 1$ (see [C], Théorème 1$)$, and on some explicit calculations involving the diagonal map.

Remark 2.4. By definition, $B(n+2, n)=8$ for all $n \geq 1$. If $\sigma^{*}$ is the cohomology suspension

$$
H^{n+3}(K(G, n+1) ; H) \rightarrow H^{n+2}(K(G, n) ; H)
$$

for any positive integer $n$ and any abelian groups $G$ and $H$, it is possible to get a better result than the one given by Theorem 2.3. In fact one can prove that

$$
2 \operatorname{im}\left(\sigma^{*}\right)=0
$$

in this special case. The result is clear for $n \geq 2$ because we have $2 H^{n+3}(K(G, n+1) ; H)=0$ since $H_{n+2}(K(G, n+1) ; \mathbb{Z})=0$ if $n \geq 1$ and 
$H_{n+3}(K(G, n+1) ; \mathbb{Z})=G \otimes \mathbb{Z} / 2$ if $n \geq 2$ (see [WH], p. 81, and [WG2], p. 560, Theorem 3.20). In order to get the result for $n=1$, we may apply the argument of the proof of the main theorem of [P2]. Consider the composition of homomorphisms

$$
H^{4}(K(G, 2) \wedge K(G, 2) ; H) \stackrel{d^{*}}{\longrightarrow} H^{4}(K(G, 2) ; H) \stackrel{\sigma^{*}}{\longrightarrow} H^{3}(K(G, 1) ; H),
$$

where $d^{*}$ is induced by the diagonal map. The group $H_{4}(K(G, 2) ; \mathbb{Z})$ can be computed by means of Whitehead's quadratic functor $\Gamma$ (see [WH], Theorem 14) or Cartan's methods (see [C], Théorème 1): it is for instance known that $H_{4}\left(K\left(\mathbb{Z} / 2^{f}, 2\right) ; \mathbb{Z}\right) \cong \mathbb{Z} / 2^{f+1}$ for all $f \geq 1$. This calculation enables us to replace Lemma 6 of [P2] by the assertion that $2 H^{4}(K(G, 2) ; H) \subset \operatorname{im}\left(d^{*}\right)$. Because $\sigma^{*} d^{*}=0$ (see [WG2], p. 383), this finally implies that $2 \operatorname{im}\left(\sigma^{*}\right)=0$.

3. The first $k$-invariant of an $\mathbf{H}$-space. In this section, we show how it is possible to deduce from Theorem 2.3 a universal upper bound for the order of the first $k$-invariant of a loop space. As every H-space can be seen as a retract of a loop space, this also provides a universal upper bound for the order of the first $k$-invariant of an $\mathrm{H}$-space.

TheOREM 3.1. Let $X$ be an $(n-1)$-connected loop space such that $\pi_{i}(X)$ $=0$ if $n<i<m$ for some integers $m>n \geq 1$. Then its first $k$-invariant $k^{m+1}(X) \in H^{m+1}\left(X[m-1] ; \pi_{m} X\right)$ satisfies

$$
B(m+1, n) k^{m+1}(X)=0 .
$$

Proof. The first $k$-invariant of $X$ is $k^{m+1}(X) \in H^{m+1}\left(X[m-1] ; \pi_{m} X\right)$ $=H^{m+1}\left(K\left(\pi_{n} X, n\right) ; \pi_{m} X\right)$. According to Proposition 2.1, it lies in the image of the cohomology suspension

$$
\sigma^{*}: H^{m+2}\left(K\left(\pi_{n} X, n+1\right) ; \pi_{m} X\right) \rightarrow H^{m+1}\left(K\left(\pi_{n} X, n\right) ; \pi_{m} X\right),
$$

which has finite exponent dividing $B(m+1, n)$ by Theorem 2.3.

For the $k$-invariant $k^{n+2}(X)$ of an $(n-1)$-connected loop space $X$ (where $n \geq 1$ ), the result of Theorem 3.1 can be improved by considering Remark 2.4.

TheOrem 3.2. Let $X$ be an $(n-1)$-connected loop space (with $n \geq 1)$. Then

$$
2 k^{n+2}(X)=0 .
$$

Remember that the James reduced product construction allows one to show that a connected CW-complex $X$ admits an H-space structure if and only if the canonical map $X \rightarrow \Omega \Sigma X$ has a left homotopy splitting (see [J]). This enables us to apply Theorem 3.2 in order to get an upper bound for the order of the first $k$-invariant of an $\mathrm{H}$-space. 
Corollary 3.3. The first $k$-invariant $k^{n+2}(X)$ of an $(n-1)$-connected $H$-space $X$ (with $n \geq 1)$ fulfills

$$
2 k^{n+2}(X)=0 .
$$

Pr o o f. Let $i: X \rightarrow \Omega \Sigma X$ denote the canonical map given by the adjoint of the identity in $[\Sigma X, \Sigma X]$. The naturality of $k$-invariants with respect to maps provides the following relation between the $k$-invariants of $X$ and those of $\Omega \Sigma X$ (see [WG2], p. 424, Theorem 2.6). Consider the homomorphism

$$
i^{*}: H^{n+2}\left(\Omega \Sigma X[n] ; \pi_{n+1} \Omega \Sigma X\right) \rightarrow H^{n+2}\left(X[n] ; \pi_{n+1} \Omega \Sigma X\right)
$$

and the coefficient homomorphism

$$
i_{*}: H^{n+2}\left(X[n] ; \pi_{n+1} X\right) \rightarrow H^{n+2}\left(X[n] ; \pi_{n+1} \Omega \Sigma X\right)
$$

induced by the map $i$. We then have

$$
i_{*}\left(k^{n+2}(X)\right)=i^{*}\left(k^{n+2}(\Omega \Sigma X)\right) .
$$

The loop space $\Omega \Sigma X$ is $(n-1)$-connected, and so it follows from Theorem 3.2 that $2 k^{n+2}(\Omega \Sigma X)=0$. As $X$ is supposed to be an H-space, $i$ has a left homotopy inverse and $i_{*}$ is a split monomorphism. Therefore, we have $2 k^{n+2}(X)=0$.

Notice that the results of this section hold for loop spaces and H-spaces even if they are not of finite type. Therefore Corollary 3.3 is a generalization of Arkowitz-Curjel's theorem for the first $k$-invariant of H-spaces. Additionally, it provides a universal upper bound for its order.

4. The order of higher $k$-invariants of iterated loop spaces. If one wants to generalize Theorem 3.2 or Corollary 3.3 to higher $k$-invariants, one has to assume that the space we are looking at is an iterated loop space. It is quite easy to check that our argument implies inductively the existence of a universal upper bound for the order of $k^{m+1}(X)$ for all $(n-1)$ connected $r$-fold loop spaces $X$, where $r=m-n$ (see Théorème 4.18 and Corollaire 4.19 of [P1]). However, since we want to prove finiteness results for the order of $k$-invariants in the unstable range, we would like to assume that $r \leq m-2 n+1$ and therefore the only interesting case would be $n=1$. The purpose of this section is to modify our method in order to get results on $k^{m+1}(X)$ for $(n-1)$-connected $r$-fold loop spaces $X$ with $r$ as small as possible. Observe that throughout this section, the spaces we consider are again not necessarily of finite type.

Definition 4.1. For $k \geq 1$, let $L_{k}$ denote the product of all prime numbers $p$ such that there exists a sequence $\left(a_{1}, a_{2}, \ldots\right)$ of non-negative integers with

(a) $a_{1} \equiv 0 \bmod 2 p-2, a_{i} \equiv 1$ or $0 \bmod 2 p-2$ for $i \geq 2$, 
(b) $a_{i} \geq p a_{i+1}$ for $i \geq 1$,

(c) $\sum_{i=1}^{\infty} a_{i}=k$.

For example, $L_{1}=1, L_{2}=2, L_{3}=2, L_{4}=6, L_{5}=6, L_{6}=2, \ldots$ Notice that $L_{k}$ divides the product of all primes $p \leq k / 2+1$.

The integers $L_{k}$ occur in the calculation of the stable homology groups of Eilenberg-MacLane spaces.

Lemma 4.2 ([C], Théorème 2). For any abelian group $G$ and any integer $s \geq 2, L_{i-s} H_{i}(K(G, s) ; \mathbb{Z})=0$ whenever $s<i<2 s$.

Lemma 4.3. Let $X$ be an $(n-1)$-connected $C W$-complex (with $n \geq 2)$ for which there is an integer $N$ satisfying $n \leq N \leq 2 n-2$ and such that $\pi_{j} X=0$ for all $j>N$.

(a) $\prod_{s=n}^{N} L_{i-s} H_{i}(X ; \mathbb{Z})=0$ if $N<i<2 n$.

(b) $\prod_{s=n}^{N+1} L_{i-s} H^{i}(X ; H)=0$ for any abelian group $H$ if $N+1<i<2 n$.

Pr o of. For $n \leq s \leq N-1$, consider the fibration

$$
K\left(\pi_{s+1} X, s+1\right) \rightarrow X[s+1] \stackrel{\alpha_{s}}{\longrightarrow} X[s]
$$

and the associated homology exact sequence

$$
H_{i}\left(K\left(\pi_{s+1} X, s+1\right) ; \mathbb{Z}\right) \rightarrow H_{i}(X[s+1] ; \mathbb{Z}) \stackrel{\left(\alpha_{s}\right)_{*}}{\longrightarrow} H_{i}(X[s] ; \mathbb{Z})
$$

provided by the Serre spectral sequence since $i<2 n$. Notice that $X[n]=$ $K\left(\pi_{n} X, n\right)$ and $X[N]=X$. The assertion (a) then follows inductively from Lemma 4.2 and (b) is a direct consequence of the universal coefficient theorem.

Definition 4.4. For $j \geq 1$ let $R_{j}=\prod_{k=1}^{j} L_{k}$. For example, $R_{1}=1$, $R_{2}=2, R_{3}=4, R_{4}=24, R_{5}=144, R_{6}=288, \ldots$ It turns out that a prime $p$ divides $R_{j}$ if and only if $p \leq j / 2+1$. For $i \geq 1$, let us also define $\bar{R}_{i}=\prod_{j=1}^{i} R_{j}$.

The integers $R_{j}$ were introduced in [A4] in order to formulate the following result (see [A4], Theorem 1.3).

THEOREM 4.5. If $X$ is an $(n-1)$-connected $r$-fold loop space (with $n \geq 1$ and $r \geq 0)$, then

$$
R_{m-n+1} k^{m+1}(X)=0 \quad \text { for } n+1 \leq m \leq 2 n+r-2 .
$$

Let us first extend the assertions of Theorem 3.2 and Corollary 3.3 to the $k$-invariants of $(n-1)$-connected loop spaces or H-spaces up to dimension $2 n$.

TheOREM 4.6. Let $X \simeq \Omega Y$ be any $(n-1)$-connected loop space (with $n \geq 1$ ), and $m$ any integer such that $n+1 \leq m \leq 2 n$. Then the $k$-invariant 


$$
\begin{array}{r}
k^{m+1}(X) \in H^{m+1}\left(X[m-1] ; \pi_{m} X\right) \text { satisfies } \\
\qquad C_{1}(m, n) k^{m+1}(X)=0,
\end{array}
$$

where

$$
C_{1}(m, n)= \begin{cases}R_{m-n+1} & \text { if } m \leq 2 n-1, \\ B(2 n+1, n) R_{n} & \text { if } m=2 n .\end{cases}
$$

Notice that the integers $C_{1}(m, n)$ depend only on $m$ and $n$ and that a prime $p$ divides $C_{1}(m, n)$ if and only if $p \leq(m-n+3) / 2$.

Proof. For $m \leq 2 n-1$, the assertion is already provided by Theorem 4.5. In order to get it for $m=2 n$, consider the fibrations

$$
\begin{aligned}
& X(n, 2 n-1] \stackrel{\beta}{\longrightarrow} X[2 n-1] \stackrel{\alpha_{n}}{\longrightarrow} K\left(\pi_{n} X, n\right), \\
& Y(n+1,2 n] \stackrel{\beta^{\prime}}{\longrightarrow} Y[2 n] \stackrel{\alpha_{n+1}^{\prime}}{\longrightarrow} K\left(\pi_{n} X, n+1\right)
\end{aligned}
$$

where $X(n, 2 n-1]$ and $Y(n+1,2 n]$ denote the $n$-connected cover of $X[2 n-1]$ and the $(n+1)$-connected cover of $Y[2 n]$ respectively. Denote by $H$ the group $\pi_{2 n} X$ and look at the induced commutative diagram

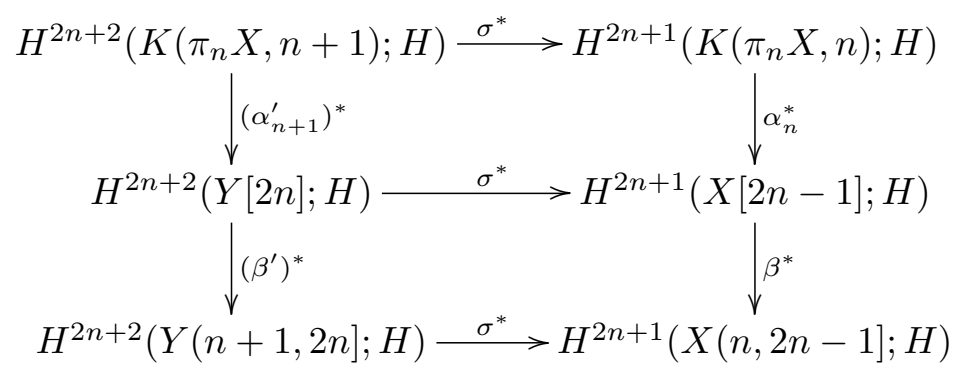

where the horizontal arrows are cohomology suspensions and where the left vertical sequence is exact. It follows from Lemma 4.3(b) that

$$
R_{n} H^{2 n+2}(Y(n+1,2 n] ; H)=0
$$

and from Theorem 2.3 that the exponent of the image of the top suspension $\sigma^{*}$ divides the integer $B(2 n+1, n)$. Consequently, the image of $\sigma^{*}: H^{2 n+2}(Y[2 n] ; H) \rightarrow H^{2 n+1}(X[2 n-1] ; H)$ is of finite exponent dividing $B(2 n+1, n) R_{n}$ and Proposition 2.1 completes the proof.

REMARK 4.7. If $n=1$, the statement of Theorem 4.6 is actually given by Theorem 3.1 but can be improved as in Theorem 3.2.

The argument of the proof of Corollary 3.3 produces also the following result which gives a positive answer in dimensions $\leq 2 n$ to Questions (A) and $(\mathrm{B})$ for $(n-1)$-connected H-spaces.

Corollary 4.8. For any $(n-1)$-connected $H$-space $X$ (with $n \geq 1)$ and any integer $m$ such that $n+1 \leq m \leq 2 n$, the $k$-invariant $k^{m+1}(X) \in$ 
$H^{m+1}\left(X[m-1] ; \pi_{m} X\right)$ fulfills

$$
C_{1}(m, n) k^{m+1}(X)=0 .
$$

In order to obtain similar results for $k$-invariants in dimensions $>2 n$, we need to assume that $X$ is an iterated loop space. Let us start by looking at double loop spaces.

Proposition 4.9. Let $X \simeq \Omega^{2} Y$ be any $(n-1)$-connected double loop space (with $n \geq 1$ ), and $m$ and $t$ positive integers such that $1 \leq t \leq n$ and $m \geq n+t+2$. Let $W$ be the loop space of $Y$ and suppose the order $\varrho_{j}(W)$ of the $k$-invariant $k^{j+1}(W)$ in the cohomology group $H^{j+1}\left(W[j-1] ; \pi_{j} W\right)$ is finite whenever $n+2 \leq j \leq m-t$. Then the order of the $k$-invariant $k^{m+1}(X) \in H^{m+1}\left(X[m-1] ; \pi_{m} X\right)$ of $X$ divides the product

$$
\varrho_{n+2}(W) \varrho_{n+3}(W) \ldots \varrho_{m-t}(W) B(m+1, n) R_{t+1} .
$$

Pr o of. Consider the fibrations

$$
\begin{aligned}
X(m-t-1, m-1] & \stackrel{\beta}{\rightarrow} X[m-1] \stackrel{\alpha_{m-t-1}}{\longrightarrow} X[m-t-1], \\
W(m-t, m] & \stackrel{\beta^{\prime}}{\rightarrow} W[m] \stackrel{\alpha_{m-t}^{\prime}}{\longrightarrow} W[m-t], \\
Y(m-t+1, m+1] & \stackrel{\beta^{\prime \prime}}{\rightarrow} Y[m+1] \stackrel{\alpha_{m-t+1}^{\prime \prime}}{\longrightarrow} Y[m-t+1],
\end{aligned}
$$

and the induced commutative diagrams

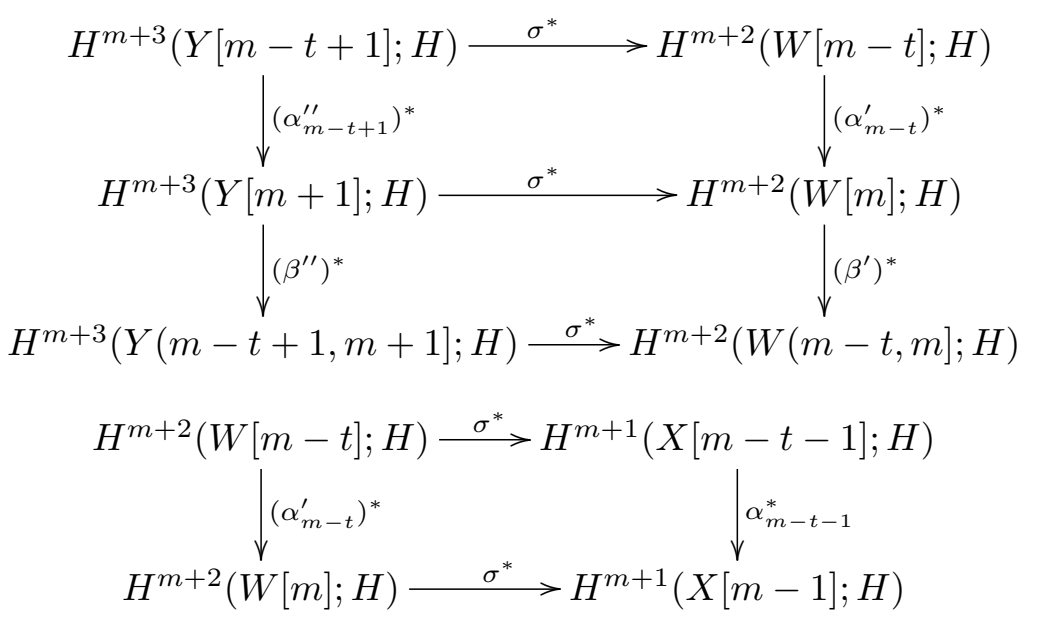

where the horizontal arrows are cohomology suspensions and where $H$ is written for the group $\pi_{m} X$. The $k$-invariant we are looking at is $k^{m+1}(X) \in$ $H^{m+1}(X[m-1] ; H)$, which is related to the corresponding $k$-invariant $k^{m+3}(Y) \in H^{m+3}(Y[m+1] ; H)$ by the formula

$$
\left(\sigma^{*}\right)^{2}\left(k^{m+3}(Y)\right)=k^{m+1}(X)
$$


because of Proposition 2.1. The left vertical sequence in the first diagram is exact since $t \leq n$. It follows from Lemma 4.3(b) that

$$
R_{t+1} H^{m+3}(Y(m-t+1, m+1] ; H)=0
$$

since $m \geq 2 t$. Consequently, the element $\left(\beta^{\prime \prime}\right)^{*}\left(R_{t+1} k^{m+3}(Y)\right)$ vanishes and there exists an element $x \in H^{m+3}(Y[m-t+1] ; H)$ such that $\left(\alpha_{m-t+1}^{\prime \prime}\right)^{*}(x)=$ $R_{t+1} k^{m+3}(Y)$. Therefore,

$$
\begin{aligned}
R_{t+1} k^{m+1}(X) & =R_{t+1}\left(\sigma^{*}\right)^{2}\left(k^{m+3}(Y)\right)=\left(\sigma^{*}\right)^{2}\left(\alpha_{m-t+1}^{\prime \prime}\right)^{*}(x) \\
& =\alpha_{m-t-1}^{*}\left(\sigma^{*}\right)^{2}(x) .
\end{aligned}
$$

According to Proposition 1.2, there are maps

$$
W[m-t] \stackrel{\varphi}{\rightarrow} \prod_{i=n+1}^{m-t} K\left(\pi_{i} W, i\right) \stackrel{\psi}{\rightarrow} W[m-t]
$$

such that the composition $\psi \varphi$ is exactly the power map $\chi^{\varrho}: W[m-t] \rightarrow$ $W[m-t]$, where $\varrho$ denotes the product $\varrho_{n+2}(W) \varrho_{n+3}(W) \ldots \varrho_{m-t}(W)$. The maps $\varphi$ and $\psi$ induce the commutative diagram

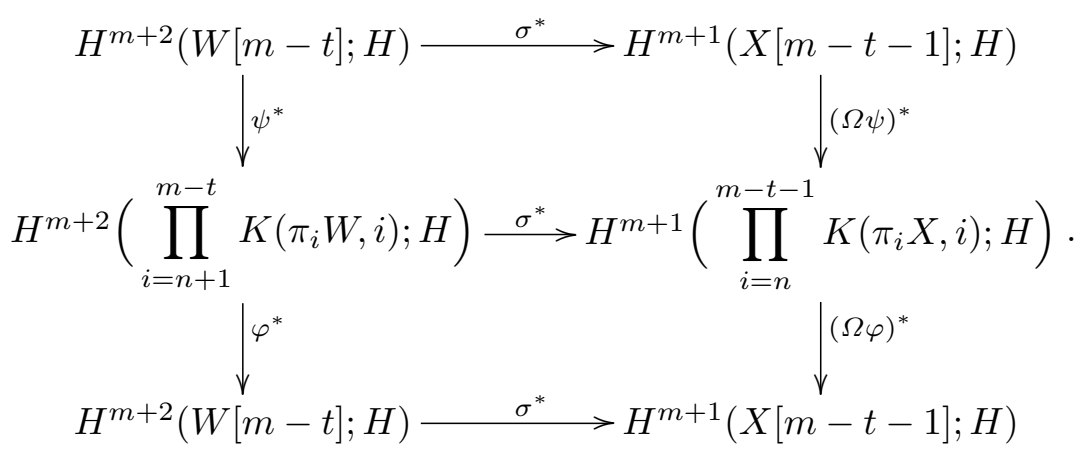

The composition $\varphi^{*} \psi^{*}$ induced by the power map $\chi^{\varrho}$ acts on primitive cohomology classes by multiplication by $\varrho$, in particular on the class $\sigma^{*}(x) \in$ $H^{m+2}(W[m-t] ; H)$. Thus, one gets $\varphi^{*} \psi^{*}\left(\sigma^{*}(x)\right)=\varrho \sigma^{*}(x)$ and

$\varrho\left(\sigma^{*}\right)^{2}(x)=\sigma^{*} \varphi^{*} \psi^{*}\left(\sigma^{*}(x)\right)=(\Omega \varphi)^{*} \sigma^{*} \psi^{*} \sigma^{*}(x) \in H^{m+1}(X[m-t-1] ; H)$.

On the other hand, we know from Theorem 2.3 that the image of the middle horizontal cohomology suspension is killed by $B(m+1, n)$. This enables us to deduce that $\varrho B(m+1, n)\left(\sigma^{*}\right)^{2}(x)=0$ and finally that

$$
\varrho B(m+1, n) R_{t+1} k^{m+1}(X)=\alpha_{m-t-1}^{*}\left(\varrho B(m+1, n)\left(\sigma^{*}\right)^{2}(x)\right)=0 .
$$

TheOREM 4.10. Let $X \simeq \Omega^{2} Y$ be any $(n-1)$-connected double loop space (with $n \geq 1$ ) and $m$ any integer such that $n+1 \leq m \leq 3 n+2$. Then the $k$-invariant $k^{m+1}(X) \in H^{m+1}\left(X[m-1] ; \pi_{m} X\right)$ satisfies

$$
C_{2}(m, n) k^{m+1}(X)=0
$$


where the integers $C_{2}(m, n)$ are given by

$$
C_{2}(m, n)= \begin{cases}R_{m-n+1} & \text { if } n+1 \leq m \leq 2 n, \\ 2 B(m+1, n) R_{m-n-1} & \text { if } m=2 n+1 \text { or } m=2 n+2, \\ B(2 n+3, n+1) B(m+1, n) \bar{R}_{n+1} R_{n+1} R_{m-2 n-1} & \text { if } 2 n+3 \leq m \leq 3 n+2 .\end{cases}
$$

Observe that $C_{2}(m, n)$ depends only on $m$ and $n$ and that a prime $p$ divides $C_{2}(m, n)$ if and only if $p \leq(m-n+3) / 2$.

Proof. If $n+1 \leq m \leq 2 n$, then the assertion is given by Theorem 4.5. If $m=2 n+1$ (and $n \geq 2$ ) or $m=2 n+2$, one has $m \geq n+3$ and one can use Proposition 4.9 with $t=m-n-2$; this shows that

$$
\varrho_{n+2}(W) B(m+1, n) R_{m-n-1} k^{m+1}(X)=0 .
$$

However, since $\varrho_{n+2}(W)$ is the order of the first $k$-invariant $k^{n+3}(W)$ of the $n$-connected space $W=\Omega Y$, it divides 2 , according to Theorem 3.2. We then obtain

$$
2 B(m+1, n) R_{m-n-1} k^{m+1}(X)=0 .
$$

If $n=1$ and $m=3$, one can also apply the idea of the proof of Proposition 4.9. We start with the fibration

$$
K\left(\pi_{2} X, 2\right) \rightarrow X[2] \rightarrow K\left(\pi_{1} X, 1\right)
$$

and consider the commutative diagram

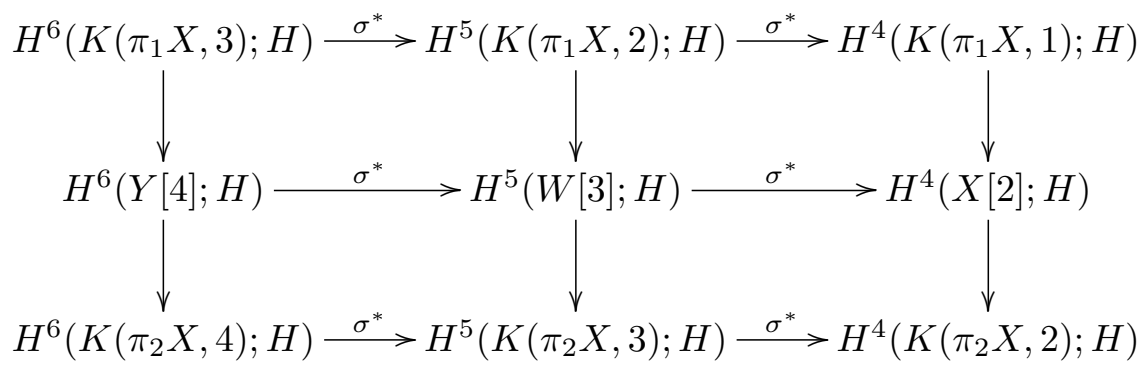

where $W=\Omega Y, H=\pi_{3} X$ and in which the left vertical sequence is exact. Again, $k^{4}(X)=\left(\sigma^{*}\right)^{2}\left(k^{6}(Y)\right) \in H^{4}(X[2] ; H)$ by Proposition 2.1. Since we know from Lemma 4.2 that $2 H^{6}\left(K\left(\pi_{2} X, 4\right) ; H\right)=0$ and from Theorem 2.3 that $B(4,1)$ kills the image of the top right cohomology suspension $\sigma^{*}$, we conclude that $2 B(4,1) k^{4}(X)=0$ (notice that $R_{1}=1$ ).

For $2 n+3 \leq m \leq 3 n+2$ choose $t=m-2 n-2$ in Proposition 4.9 to deduce that

$$
\varrho_{n+2}(W) \varrho_{n+3}(W) \ldots \varrho_{2 n+2}(W) B(m+1, n) R_{m-2 n-1} k^{m+1}(X)=0 .
$$

Since $W$ is an $n$-connected loop space, Theorem 4.6 implies that $\bar{R}_{n+1}$ is a positive multiple of the product $\varrho_{n+2}(W) \varrho_{n+3}(W) \ldots \varrho_{2 n+1}(W)$ and that 
$\varrho_{2 n+2}(W)$ divides $B(2 n+3, n+1) R_{n+1}$. Consequently, we get

$$
B(2 n+3, n+1) B(m+1, n) \bar{R}_{n+1} R_{n+1} R_{m-2 n-1} k^{m+1}(X)=0 .
$$

In the general case, we are able to prove the following solution of Questions (A) and (B).

TheOREM 4.11. Let $X$ be any $(n-1)$-connected $r$-fold loop space (with $n \geq 1$ and $r \geq 2$ ) and $m$ any integer such that $n+1 \leq m \leq(r+1) n+r$. Then the $k$-invariant $k^{m+1}(X) \in H^{m+1}\left(X[m-1] ; \pi_{m} X\right)$ satisfies

$$
C_{r}(m, n) k^{m+1}(X)=0
$$

where the integers $C_{r}(m, n)$ are inductively defined for $r \geq 3$ by the following formulae:

$$
C_{r}(m, n)= \begin{cases}R_{m-n+1} & \text { if } n+1 \leq m \leq 2 n+r-2, \\ \left(\prod_{j=n+2}^{m-n} C_{r-1}(j, n+1)\right) B(m+1, n) R_{n+1}\end{cases}
$$

Observe that $C_{r}(m, n)$ depends only on $r, m$ and $n$ and that a prime $p$ divides $C_{r}(m, n)$ if and only if $p \leq(m-n+3) / 2$.

P r o of. If $n+1 \leq m \leq 2 n+r-2$, then the assertion follows directly from Theorem 4.5. Let us prove it for $2 n+r-1 \leq m \leq n(r+1)+r$ by induction on $r$. If $r=2$, this is given by Theorem 4.10. Now consider an $r$-fold loop space $X \simeq \Omega^{r} Y$ with $r \geq 3$ and suppose that the statement holds for all $(r-1)$-fold loop spaces. By Proposition 4.9 with $t=n$, we obtain

$$
\varrho_{n+2}(W) \varrho_{n+3}(W) \ldots \varrho_{m-n}(W) B(m+1, n) R_{n+1} k^{m+1}(X)=0,
$$

where $W$ is written for the space $\Omega^{r-1} Y$. Since $W$ is an $n$-connected $(r-1)$ fold loop space, we can deduce from the induction hypothesis that $\varrho_{j}(W)$ divides $C_{r-1}(j, n+1)$ for $n+2 \leq j \leq m-n$, because $m-n \leq r n+2 r-1$. Consequently,

$$
\left(\prod_{j=n+2}^{m-n} C_{r-1}(j, n+1)\right) B(m+1, n) R_{n+1} k^{m+1}(X)=0 .
$$

There is another way to formulate Theorems 4.6, 4.10 and 4.11.

Corollary 4.12. Let $X$ be any $(n-1)$-connected $C W$-complex and $m$ any integer $\geq n+1$. There are integers $D(m, n)$, depending only on $m$ and $n$ and divisible exactly by the primes $p \leq(m-n+3) / 2$, such that if one assumes that

$$
X \simeq \begin{cases}\Omega^{[m /(n+1)]} Y & \text { if } m \neq 2 n+1, \\ \Omega^{2} Y & \text { if } m=2 n+1,\end{cases}
$$


for some $C W$-complex $Y$, then the $k$-invariant $k^{m+1}(X)$ fulfills

$$
D(m, n) k^{m+1}(X)=0 .
$$

Proof. Take $D(m, n)=C_{1}(m, n)$ if $n+1 \leq m \leq 2 n$, respectively $D(m, n)=C_{2}(m, n)$ if $2 n+1 \leq m \leq 3 n+2$, and apply Theorem 4.6, respectively Theorem 4.10. When $m \geq 3 n+3$, the result is produced by Theorem 4.11 by setting $D(m, n)=C_{[m /(n+1)]}(m, n)$.

REMARK 4.13. It is possible to improve these results, i.e., to decrease the number of iterated loops, if one assumes that some homotopy groups $\pi_{j} X$ (for $n<j<m$ ) vanish (see [P1], Théorème 4.28).

5. The order of the identity. Our aim in this last section is to apply some of the results of Sections 3 and 4 in order to obtain an estimate for the order of the identity of H-spaces and iterated loop spaces. Some of the results stated below were suggested by F. R. Cohen.

Definition 5.1. Let $X$ be a homotopy associative H-space. We define the order of the identity of $X$ to be the order of the homotopy class of the identity $X \rightarrow X$ in $[X, X]$. The order of the identity of $X$ can be described as the least integer $s>0$ such that the $s$ th power map $\chi^{s}: X \rightarrow X$ satisfies $\chi^{s} \simeq *$. This is sometimes also called the multiplicative exponent of $X$.

As $\chi^{s}$ induces multiplication by $s$ on homotopy groups, we deduce that if $X$ is an $\mathrm{H}$-space with multiplicative exponent $s$, then all homotopy groups of $X$ are of finite exponent dividing $s$. In the following, we are interested in getting a kind of converse of this statement. This is easy to obtain for Eilenberg-MacLane spaces.

Lemma 5.2. Let $G$ be an abelian group of finite exponent $s$ and $n$ an integer $\geq 1$. Then the identity of $K(G, n)$ is of order $s$. If $G_{1}, \ldots, G_{m}$ are abelian groups with finite exponents $s_{1}, \ldots, s_{m}$, then the order of the identity of $\prod_{i=1}^{m} K\left(G_{i}, i\right)$ equals $\operatorname{lcm}\left(s_{1}, \ldots, s_{m}\right)$.

Proof. This is clear since $[K(G, n), K(G, n)] \cong H^{n}(K(G, n) ; G)$.

One can generalize this to H-spaces having a finite number of non-trivial homotopy groups in the following way.

Proposition 5.3. Let $X$ be a homotopy associative H-space such that $\pi_{i} X$ is of finite exponent $s_{i}$ for $1 \leq i \leq m$. Then the order of the identity of $X[m]$ divides $\prod_{i=1}^{m} s_{i}$.

Pr o of. Suppose by induction that the order of the identity of $X[m-1]$ divides $\prod_{i=1}^{m-1} s_{i}$ and consider the following diagram, which commutes up to 
homotopy:

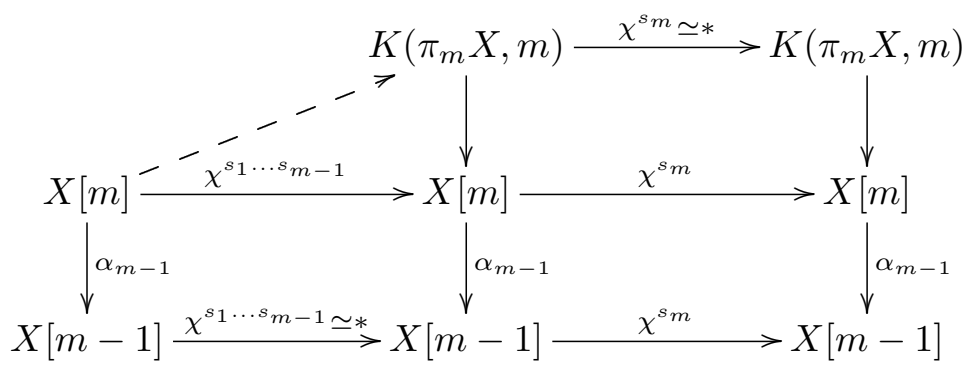

where $\alpha_{m-1}$ denotes the $(m-1)$ st Postnikov section of $X[m]$. As $\chi^{s_{1} \ldots s_{m-1}}$ : $X[m] \rightarrow X[m]$ factors through $K\left(\pi_{m} X, m\right)$ and $\chi^{s_{m}} \simeq *$ on $K\left(\pi_{m} X, m\right)$ by Lemma 5.2, we conclude that $\chi^{s_{1} \ldots s_{m}} \simeq *$ on $X[m]$.

Note that the latter argument only requires knowledge of the homotopy groups of $X$ and fails to use further information on $X$. Therefore the upper bound for the order of the identity of $X[\mathrm{~m}]$ given in Proposition 5.3 may not be the best possible (see the second statement of Lemma 5.2 for instance). As we show in the next theorem, the multiplicative exponent of the Postnikov section $X[m]$ can be given an upper bound in terms of the exponents of the homotopy groups $\pi_{i} X$ for $1 \leq i \leq m$ and of the orders of the $k$-invariants $k^{i+1}(X)$ for $2 \leq i \leq m$ when assuming all of them finite.

TheOrem 5.4. Let $X$ be a homotopy associative $H$-space and $m \geq 1$ and integer. Assume that $\pi_{i} X$ is of finite exponent $s_{i}$ for $1 \leq i \leq m$ and that $k^{i+1}(X)$ is of finite order $\varrho_{i}(X)$ for $2 \leq i \leq m$. Then the order of the identity of $X[m]$ divides the product

$$
\operatorname{lcm}\left(s_{1}, \ldots, s_{m}\right) \prod_{i=2}^{m} \varrho_{i}(X) .
$$

Proof. Let

$$
s=\operatorname{lcm}\left(s_{1}, \ldots, s_{m}\right) \quad \text { and } \quad \varrho=\prod_{i=2}^{m} \varrho_{i}(X) .
$$

According to Proposition 1.2 there are maps

$$
\varphi_{m}: X[m] \rightarrow \prod_{i=1}^{m} K\left(\pi_{i} X, i\right) \quad \text { and } \quad \psi_{m}: \prod_{i=1}^{m} K\left(\pi_{i} X, i\right) \rightarrow X[m]
$$

such that $\psi_{m} \varphi_{m} \simeq \chi^{\varrho}: X[m] \rightarrow X[m]$. As $\varphi_{m}$ can be shown to be an H-map, we may look at the following commutative diagram: 


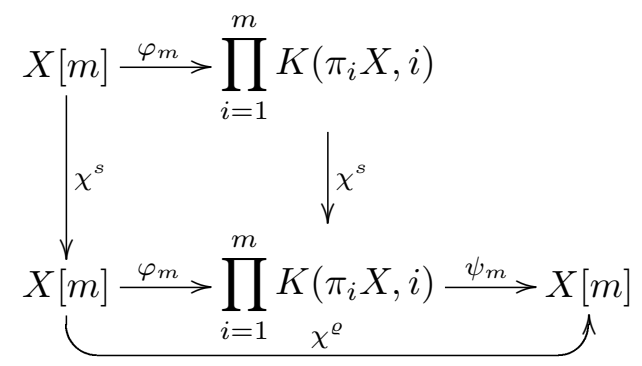

and we get $\chi^{s \varrho} \simeq \chi^{\varrho} \chi^{s} \simeq \psi_{m} \varphi_{m} \chi^{s} \simeq \psi_{m} \chi^{s} \varphi_{m} \simeq *$ because the order of the identity of $\prod_{i=1}^{m} K\left(\pi_{i} X, i\right)$ divides $s$ by Lemma 5.2 .

We can now use the upper bounds for the order of $k$-invariants of $\mathrm{H}$ spaces and iterated loop spaces obtained in Corollary 4.8 and Theorem 4.11 to give an estimate for the order of the identity of certain Postnikov sections of H-spaces and iterated loop spaces.

COROLlaRY 5.5. Let $X$ be an $(n-1)$-connected homotopy associative $H$-space (with $n \geq 1$ ), $m$ an integer satisfying $n+1 \leq m \leq 2 n$, and assume that $\pi_{i} X$ has finite exponent $s_{i}$ for $n \leq i \leq m$. Then the order of the identity of $X[m]$ divides the product

$$
\operatorname{lcm}\left(s_{n}, \ldots, s_{m}\right) \prod_{i=n+1}^{m} C_{1}(i, n),
$$

where the integers $C_{1}(i, n)$ were defined in Theorem 4.6.

This shows for example that if the homotopy groups $\pi_{n} X$ and $\pi_{n+1} X$ of an $(n-1)$-connected H-space $X$ have finite exponents $s_{n}$ and $s_{n+1}$, then the order of the identity of $X[n+1]$ divides $2 \operatorname{lcm}\left(s_{n}, s_{n+1}\right)$.

Corollary 5.6. Let $X$ be an $(n-1)$-connected $r$-fold loop space (with $n \geq 1$ and $r \geq 2), m$ an integer satisfying $n+1 \leq m \leq(r+1) n+r$, and assume that $\pi_{i} X$ has finite exponent $s_{i}$ for $n \leq i \leq m$. Then the order of the identity of $X[\mathrm{~m}]$ divides the product

$$
\operatorname{lcm}\left(s_{n}, \ldots, s_{m}\right) \prod_{i=n+1}^{m} C_{r}(i, n),
$$

where the integers $C_{r}(i, n)$ were defined in Theorems 4.10 and 4.11 .

REMARK 5.7. The above results on the order of the identity of some $X[m]$ can be generalized in an easy way to any element of $[Y, X[m]]$, where $Y$ is an arbitrary $\mathrm{CW}$-complex. It suffices to notice that $f \in[Y, X[m]]$ factors as $\operatorname{id}_{X[m]} f$, where $\operatorname{id}_{X[m]}$ denotes the class of the identity of $X[m]$. 


\section{References}

[AC] M. Arkowitz and C. Curjel, The Hurewicz homomorphism and finite homotopy invariants, Trans. Amer. Math. Soc. 110 (1964), 538-551.

[A1] D. Arlettaz, On the homology of the special linear group over a number field, Comment. Math. Helv. 61 (1986), 556-564.

[A2] - On the $k$-invariants of iterated loop spaces, Proc. Roy. Soc. Edinburgh Sect. A 110 (1988), 343-350.

[A3] - , The first k-invariant of a double loop space is trivial, Arch. Math. (Basel) 54 (1990), 84-92.

[A4] -, Universal bounds for the exponent of stable homotopy groups, Topology Appl. 38 (1991), 255-261.

[A5] —, Exponents for extraordinary homology groups, Comment. Math. Helv. 68 (1993), 653-672.

[C] H. Cartan, Algèbres d'Eilenberg-MacLane et homotopie, Séminaire H. Cartan Ecole Norm. Sup. (1954/1955), exp. 11; see also: Oeuvres, Vol. III, Springer, 1979, 1374-1394.

[J] I. M. James, Reduced product spaces, Ann. of Math. (2) 62 (1955), 170-197.

[P1] N. Pointet-Tischler, Invariants de Postnikov des espaces de lacets, thèse, Université de Lausanne, 1996.

[P2] - La suspension cohomologique des espaces d'Eilenberg-MacLane, C. R. Acad. Sci. Paris Sér. I 325 (1997), 1113-1116.

[Sm] L. Smith, On the relation between spherical and primitive homology classes in topological groups, Topology 8 (1969), 69-88.

[So] C. Soulé, Opérations en K-théorie algébrique, Canad. J. Math. 37 (1985), 488550 .

[T] R. Thom, L'homologie des espaces fonctionnels, in: Colloque de topologie algébrique (Louvain, 1956), Masson, 1957, 29-39.

[WG1] G. Whitehead, On spaces with vanishing low-dimensional homotopy groups, Proc. Nat. Acad. Sci. U.S.A. 34 (1948), 207-211.

[WG2] - Elements of Homotopy Theory, Grad. Texts in Math. 61, Springer, 1978.

[WH] J. H. C. Whitehead, A certain exact sequence, Ann. of Math. (2) 52 (1950), 51-110.

Institut de mathématiques

Université de Lausanne

CH-1015 Lausanne, Switzerland

E-mail: dominique.arlettaz@ima.unil.ch
Max-Planck-Institut für Mathematik

D-53072 Bonn, Germany

Current address (N. Pointet-Tischler): Zelgstrasse 44

CH-8134 Adliswil, Switzerland E-mail: npointet@datacomm.ch 